\title{
Cops and Robbers playing on edges
}

\author{
Andrzej Dudek*, Przemyseaw Gordinowicz, and Pawę PraŁat ${ }^{\dagger}$
}

In the game of cops and robbers, the cops try to capture a robber moving on the vertices of the graph. The minimum number of cops required to win on a given graph $G$ is called the cop number of $G$. In this paper, we consider the variant of the game in which both players play on edges instead of vertices, yielding the edge cop number. We relate the new graph parameter to the classic one, investigate Meyniel extremal families, and characterize edge copwin graphs. We also play the game on random graphs and planar graphs.

\section{Introduction}

The game of Cops and Robbers, introduced independently by Nowakowski and Winkler [23] and Quilliot [28] thirty years ago, is played on a fixed graph $G$. We will always assume that $G$ is undirected, simple, and finite. There are two players, a set of $k$ cops, where $k \geq 1$ is a fixed integer, and the robber. The cops begin the game by occupying any set of $k$ vertices (in fact, for a connected $G$, their initial position does not matter). The robber then chooses a vertex, and the cops and robber move in alternate rounds. The players use edges to move from vertex to vertex. More than one cop is allowed to occupy a vertex, and the players may remain on their current positions. The players know each other's current locations. The cops win and the game ends if at least one of the cops eventually occupies the same vertex as the robber; otherwise, that is, if the robber can avoid this indefinitely, he wins. As placing a cop on each vertex guarantees that the cops win, we may define the cop number, written $c(G)$, which is the minimum number of cops needed to win on $G$. The cop number was introduced by Aigner and Fromme [1] who proved (among other things) that if $G$ is connected and planar, then $c(G) \leq 3$. For more results on vertex pursuit games such as Cops

*The first author is supported in part by Simons Foundation Grant \#244712 and by a grant from the Faculty Research and Creative Activities Award (FRACAA), Western Michigan University.

†The third author is supported in part by NSERC and Ryerson University. 
and Robbers, the reader is directed to the surveys on the subject $[3,14,17]$ and recent monograph [10].

Suppose now that both players are slightly bored and, in order to make the game more interesting, they have decided to play on edges instead of vertices. In other words, the players start the game on edges and use vertices to move from edges to edges. The other rules of the game remain unchanged. We define the edge cop number, $\bar{c}(G)$, to be the counterpart of the cop number for the classic game. It is clear that playing the new game on a graph $G$ is equivalent to playing the classic game on $L(G)$, the line graph of $G$; that is, $\bar{c}(G)=c(L(G))$. However, it seems that it is not beneficial to look at the problem this way, especially if one is interested in discovering properties of the new graph parameter and trying to relate it to some other parameters, including the classic cop number. For example, as we already mentioned $c(G) \leq 3$ for any connected, planar graph $G$ but, since $L(G)$ is usually not planar, we cannot say anything about $\bar{c}(G)$. However, by playing on edges of $G$ and using the planarity of this graph one can show that the bound of 3 still holds. The only proof in this paper that uses the fact that $\bar{c}(G)=c(L(G))$ is the proof of Lemma 3.3.

In Section 2, we relate the edge cop number to the classic one. We characterize graphs with the edge cop number equal to one in Section 3. In Section 4, we investigate Meyniel extremal families. In particular, the edge cop number of incidence graphs of the projective plane is studied. Random graphs are considered in Section 5 and planar graphs in Section 6. We conclude the paper with some open problems and future directions that can be found in Section 7.

\section{Relation to the cop number}

In this section, we relate the edge cop number $\bar{c}(G)$ to the classic cop number $c(G)$.

Theorem 2.1. Let $G$ be any connected graph. Then

$$
\left\lceil\frac{c(G)}{2}\right\rceil \leq \bar{c}(G) \leq c(G)+1
$$

Before we move to the proof of this theorem, let us mention that this result is sharp. For the upper bound, note that for $n \geq 4, \bar{c}\left(K_{n}\right)=2$ whereas $c\left(K_{n}\right)=1$. (Characterization of graphs with $\bar{c}(G)=c(G)+1$ is left as an open problem - see Section 7 for more details.) For the lower bound, one can check that the Petersen graph (or the graph presented in Figure 1) has 


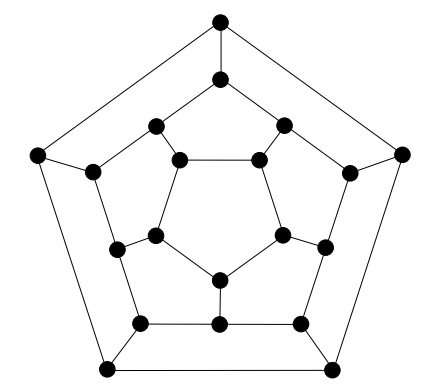

Figure 1: A plane representation of the dodecahedron.

the classic and the edge cop number of 3 and of 2, respectively, yielding $\left\lceil\frac{3}{2}\right\rceil=2$. (Having said that, we note that a small improvement may be possible and perhaps $\lceil(c(G)+1) / 2\rceil \leq \bar{c}(G)$ - see Section 7 for more details.) More importantly, we will show (Theorem 5.1) that asymptotically almost surely the ratio $\bar{c}(\mathcal{G}(n, p)) / c(\mathcal{G}(n, p))$ is equal to $1 / 2+o(1)$ for dense random graphs. This, in particular, implies that for almost all graphs the classic cop number is (roughly) twice as much as the edge one.

Proof of Theorem 2.1. For both inequalities that are to be proved, we are going to relate the two corresponding games: the edge variant of the game and the classic one. Hence, a winning strategy for one game can be translated to a winning strategy of the other. At any point of the game on edges and for every player occupying an edge $e=u v$ (regardless whether it is a cop or the robber), we call one of the endpoints (say, $u$ ) a head of this player and the other endpoint (say, $v$ ) is a tail. When a player makes a move, he can move from edge to edge either through a head or a tail, making sure the distances travelled by heads and tails are at most 1 . For example, when a player moves from $e=u v$ to $e^{\prime}=v w$, vertex $v$ that used to be a tail becomes a head and $w$ is a tail.

Let us start with a proof of $\bar{c}(G) \leq c(G)+1$. We play the edge variant of the game with $c+1$ cops, having in mind that $c=c(G)$ cops can win the classic variant. The players start the game as usual, the team of $c+1$ cops go first to some edges and then the robber goes to some edge. We distinguish two phases in this game. At the beginning of the first phase, we select any $c$ cops that will be actively playing during this phase; the remaining cop is passive, getting ready for the second phase. By looking at heads of all active players, we can mimic the classic game on vertices so that $c$ heads of cops are chasing the head of the robber. (Note that it might happen that a head of some player needs to move to a tail of this player. But this causes no 
problem, since we may simply swap them.) Since $G$ is connected, there is a winning strategy which guarantees that at some point a head of some cop catches the head of the robber, and the first phase ends. During the second phase, the cop that caught the head of the robber will have a special task of chasing the robber till the end of the game so that their heads are always matched. The remaining $c$ cops (including the one that was passive in the first phase), mimicking the classic game, follow their winning strategy which guarantees that a tail of some cop catches the tail of the robber. The robber is caught in the next round and the upper bound holds.

Now, we move to a proof of $c(G) \leq 2 \bar{c}(G)$. We play the classic variant of the game with $2 c$ cops, having in mind that $c=\bar{c}(G)$ cops can win the edge variant. The cops start the game by occupying edges $u_{1} v_{1}, u_{2} v_{2}, \ldots, u_{c} v_{c}$, so we send $2 c$ cops to occupy all endpoints of these edges. (It might happen that more than one cop is sent to one vertex.) The robber responds by occupying a vertex $v$. Let $u$ be any neighbour of $v$ (recall that $G$ is connected so $v$ has at least one neighbour). To couple the two games, we may assume that the robber starts the edge variant by occupying the edge $u v$. From that point on, we couple the two games as before. Since $c$ cops have a strategy to win the edge variant of the game, one of $2 c$ cops catch the robber in the classic game. The lower bound holds and the proof is finished.

\section{Characterization of edge cop-win graphs}

In this section, we investigate the special case when one cop can win the game. If $c(G)=1$ (or $\bar{c}(G)=1$ ), we say $G$ is cop-win (or edge cop-win, respectively). Nowakowski and Winkler [23] and, independently Quilliot [28], considered the game with one cop only. As we already mentioned, the introduction of the cop number came in [1].

The structure of cop-win graphs has been relatively well-understood. In $[23,28,29]$ a kind of ordering of the vertex set-now called a cop-win ordering - was introduced which completely characterizes such graphs. If $u$ is a vertex, then the closed neighbour set of $u$, written $N[u]$, consists of $u$ along with the neighbours of $u$. A vertex $u$ is a corner if there is some vertex $v, v \neq u$, such that $N[u] \subseteq N[v]$. A graph is dismantlable if some sequence of deleting corners results in the graph with a single vertex. For example, each tree is dismantlable and, more generally, so are chordal graphs (that is, graphs with no induced cycles of length more than three). To prove the latter fact, it is enough to note that a chordal graph contains a vertex (called simplicial) whose neighbour set is a clique.

The following theorem provides a characterization of cop-win graphs. 
Theorem 3.1. [23, 28, 29]

(i) If $u$ is a corner of a graph $G$, then $G$ is cop-win if and only if $G-u$ is cop-win.

(ii) A graph is cop-win if and only if it is dismantlable.

From Theorem 3.1, cop-win graphs have a recursive structure, made explicit in the following sense. A permutation $v_{1}, v_{2}, \ldots, v_{n}$ of the vertices of $G$ is a cop-win ordering if there exist vertices $w_{1}, w_{2}, \ldots, w_{n}$ such that, for all $i \in\{1,2, \ldots, n\}, N\left[v_{i}\right] \subseteq N\left[w_{i}\right]$ in $V(G) \backslash\left\{v_{j}: j<i\right\}$ and $v_{i} \neq w_{i}$. This result implies that the family of cop-win graphs is quite rich. On the other hand, it was shown by Bonato, Kemkes, and the third author that almost all cop-win graphs contain a universal vertex, that is, a vertex that is joined to all other vertices [9]. (See also [24] where this result was generalized and it was shown that almost all $k$-cop-win graphs contain a dominating set of cardinality $k$.)

In this section, we characterize edge cop-win graphs. The following theorem is the main result of this section.

Theorem 3.2. A graph $G$ is edge cop-win if and only if the following three properties hold:

(i) $G$ is connected,

(ii) $G$ is chordal,

(iii) $G$ is $K_{4}$-free.

We start with a definition of the following useful process. Let $G_{2}$ be a graph consisting of two vertices $v_{1}, v_{2}$ and a single edge $v_{1} v_{2}$. For a given integer $t \geq 3$, we construct $G_{t}$ from $G_{t-1}$ by adding one more vertex $v_{t}$ and performing a vertex step or an edge step. When a vertex step is performed, any vertex $v_{i} \in V\left(G_{t-1}\right)$ is selected and one edge $v_{i} v_{t}$ is added. When an edge step is performed, any edge $v_{i} v_{j} \in E\left(G_{t-1}\right)$ is selected and two edges $v_{i} v_{t}$ and $v_{j} v_{t}$ are added. Note that a graph $G_{t}$ consists of $t$ vertices. We say that a graph $G$ on $n$ vertices is constructible if it can be generated during this process; that is, there exists a process generating a sequence of graphs $\left(G_{t}: t \geq 2\right)$ with $G=G_{n}$.

Lemma 3.3. Graph $G$ is edge cop-win if and only if $G$ is constructible.

Proof. Consider any process $\left(G_{t}: t \geq 2\right)$. We will show first that for any $t \geq 3$ we have $\bar{c}\left(G_{t-1}\right)=1$ if and only if $\bar{c}\left(G_{t}\right)=1$. In order to prove the claim, we simply use Theorem 3.1 and use the fact that playing the game on edges of $G$ is equivalent to playing the classic game on the line graph of $G$. Suppose first that in order to get $G_{t}$ from $G_{t-1}$ a vertex step was performed. 
Let $e=v_{i} v_{t}$ be the edge added at this step and $e^{\prime}=v_{j} v_{i}$ be any other edge incident to $e$. Since $N[e] \subseteq N\left[e^{\prime}\right]$ in the line graph $L(G), e$ is a corner and the claim holds by Theorem 3.1(i). Suppose then that $G_{t}$ was created after an edge step was performed; an edge $e=v_{i} v_{j}$ was selected, and two edges $e^{\prime}=v_{i} v_{t}$ and $e^{\prime \prime}=v_{j} v_{t}$ were added. Since $N\left[e^{\prime}\right] \subseteq N[e], e^{\prime}$ is a corner in $L(G)$ ( $e^{\prime \prime}$ is also a corner) and the claims holds as well.

Using the claim, we get immediately one direction of the statement: if $G$ is constructible, then $\bar{c}(G)=1$. Hence, it remains to prove the converse. We prove it by induction on number of vertices. For the base step note than the only connected graph on 2 vertices is both edge cop-win and constructible. Let $n \in \mathbb{N}, n \geq 3$, and $G$ be any graph on $n$ vertices such that $\bar{c}(G)=1$. Assuming that every edge cop-win graph on $n-1$ vertices is constructible we need to show that $G$ is constructible. Consider the game played on $G$. The cop has a strategy to catch the robber but we assume that the robber tries to play as long as possible. What happens when the game is about to end? The robber occupies an edge $u v$ and the cop occupies an edge incident to $u v$, say, $v w$. No matter what the robber does in the next round, he will be caught by the cop and the game ends. The only two possible scenarios are: $u$ has only one neighbour, vertex $v$, or $u$ has two neighbours, $v$ and $w$. (In every other scenario, the robber could survive for another round.) No matter which situation actually occurs, $G$ can be constructed from $G-u$ by performing a vertex step or an edge step, respectively. Since $\bar{c}(G)=1$, we can use the claim one more time to get that $\bar{c}(G-u)=1$. From the assumption it follows that $G-u$ is constructible, hence $G$ is constructible as well and the proof is finished.

Theorem 3.2 follows immediately from Lemma 3.3 and the following lemma.

Lemma 3.4. A graph $G$ is constructible if and only if the following three properties hold:

(i) $G$ is connected,

(ii) $G$ is chordal,

(iii) $G$ is $K_{4}$-free.

Proof. It is an easy observation that a constructible graph $G$ satisfies the three properties. Suppose then that a graph $G$ satisfies properties (i)-(iii). Since $G$ is chordal (property (ii)), there exists a simplicial vertex $v \in V(G)$; that is, $N[v]$ induces a clique. Since $G$ is $K_{4}$-free (property (iii)), $N[v]$ is isomorphic to $K_{2}$ or $K_{3}$. It follows that $G$ can be constructed from $G-v$ by performing a vertex step or an edge step, respectively. Moreover, since $v$ 
is simplicial, $G-v$ is chordal and so we can keep repeating the argument to deconstruct $G$ ( $G$ is connected by property (i)). The result holds.

Since any connected, chordal graph is cop-win we get immediately the following corollary.

Corollary 3.5. If $G$ is edge cop-win, then $G$ is cop-win.

Moreover, every constructible graph is planar and sparse (every constructible graph $G_{n}$ on $n$ vertices has at most $2 n-1$ edges) and so every edge cop-win graph has the two properties.

\section{Meyniel's conjecture and projective planes}

The most important open problem in this area is Meyniel's conjecture (communicated by Frankl [13]). It states that $c(n)=O(\sqrt{n})$, where $c(n)$ is the maximum of $c(G)$ over all $n$-vertex connected graphs. If true, the estimate is best possible as one can construct a bipartite graph based on the finite projective plane with the cop number of order at least $\sqrt{n}$. (See below for more details.) Up until recently, the best known upper bound of $O(n \log \log n / \log n)$ was given in [13]. It took 20 years to show that $c(n)=O(n / \log n)$ as proved in [12]. Today we know that the cop number is at most $n 2^{-(1+o(1)) \sqrt{\log _{2} n}}$ (which is still $n^{1-o(1)}$ ) for any connected graph on $n$ vertices (the result obtained independently by Lu and Peng [21], Scott and Sudakov [30], and Frieze, Krivelevich, and Loh [15]).

It is believed that the conjecture is true. However, if one looks for counterexamples for Meyniel's conjecture it is natural to study first the cop number of random graphs. It was recently shown that it is impossible and, in fact, Meyniel's conjecture holds asymptotically almost surely for binomial random graphs [27] as well as random $d$-regular graphs [26]. (See the next section for more.) These results for random graph models support Meyniel's conjecture although there is currently a huge gap in the deterministic bounds: it is still not known whether there exists $\varepsilon>0$ such that the cop number of connected graphs of order $n$ is $O\left(n^{1-\varepsilon}\right)$.

In this paper, we study the edge cop number $\bar{c}(G)$. It follows from Theorem 2.1 that $c(G) / 2 \leq \bar{c}(G) \leq 2 c(G)$ for every connected graph $G$. As a result, Meyniel's conjecture for the new graph parameter $\bar{c}(G)$ is equivalent to the classic conjecture for $c(G)$. However, even if we believe the conjecture is true, the question remains how close the cop numbers can approach $\sqrt{n}$ from below. We address this question in this section. 
Following terminology that was introduced by Baird and Bonato in [4], we call an infinite family of graphs $\left(G_{n}: n \geq 1\right)$ Meyniel extremal (or $d$ Meyniel extremal) if there exists a universal constant $d$ such that for all $n$ we have $c\left(G_{n}\right) \geq d \sqrt{\left|V\left(G_{n}\right)\right|}$. As pointed out in [4], incidence graphs are very useful in constructing graphs with large cop numbers (see [8] for more). An incidence structure $S$ consists of a set $P$ of points, and a set $L$ of lines along with an incidence relation consisting of ordered pairs of points and lines. Projective planes are some of the most well-studied examples of incidence structures. A projective plane of order $q, P G(2, q)$, consists of a set $P$ of points and a set $L$ of lines satisfying the following:

- There is exactly one line incident with every pair of distinct points;

- There is exactly one point incident with every pair of distinct lines;

- Every line consists of $q+1$ points and every point lies on $q+1$ lines;

- $|P|=|L|=q^{2}+q+1$.

Given an incidence structure $S$, we define its incidence graph $G(S)$ to be the bipartite graph whose vertices consist of the points (one partite set), and lines (the second partite set), with a point joined to a line if it is incident with the line in $S$.

A girth of a graph $G$ is defined as the length of a shortest cycle. As proved in [1] by Aigner and Fromme, if the girth of $G$ is at least 5, then $c(G) \geq \delta(G)$, where $\delta(G)$ is the minimum degree of $G$. It is known (see, for example, [16]) that for every $q$ that is a prime power the projective plane $P:=P G(2, q)$ exists. Let $G(P)$ be the corresponding incidence graph. It immediately follows from the above properties of $P G(2, q)$ that $G(P)$ is connected, has girth 6 , is $(q+1)$-regular, and has $2\left(q^{2}+q+1\right)$-many vertices. Hence,

$$
c(G(P)) \geq q+1=\sqrt{\frac{2\left(q^{2}+2 q+1\right)}{2}} \geq \sqrt{\frac{|V(G(P))|}{2}},
$$

and so this family is $(1 / \sqrt{2})$-Meyniel extremal. (In fact, $c(G(P))=q+1$ as shown by the third author in [25].) It is not known if this family yields the largest constant $d$ (since Meyniel's conjecture is still open!), but no better construction has been found.

Let us now come back to the edge cop number $\bar{c}(G)$ we investigate in this paper. Meyniel extremal families for the new parameter can be defined the same way. Moreover, it follows from Theorem 2.1 that every $d$-Meyniel extremal family for the classic cop number is $(d / 2)$-Meyniel extremal for the edge cop number. In particular, the family of incidence graphs of the 
projective plane is $(1 / 2 \sqrt{2})$-Meyniel extremal. However, this time it is not clear if the constant is best possible. Our goal is to show that, in fact, this family is $(1 / \sqrt{2})$-Meyniel extremal for the edge variant of the game. As a result, this family provides the best known construction, as for the classic case.

We start with the following observation, the counterpart of the observation of Aigner and Fromme from [1] that appears to be slightly more complicated for the edge cop number.

Lemma 4.1. Let $G$ be any connected graph with girth at least 6 . Then

$$
\bar{c}(G) \geq \delta(G) .
$$

Proof. Let $G=(V, E)$ be any connected graph with girth at least 6 . We start with a few definitions. Suppose that the robber occupying an edge $u v \in E$ is not caught and that it is his turn to make a move. An edge incident to $u v$ is called bad if it is occupied by a cop (that is, the distance to a closest cop is 0 ). An edge incident to $u v$ that is not bad is called threatening if it is incident to an edge occupied by a cop (that is, the distance to a closest cop is 1). An incident edge that is neither bad nor threatening is called good (that is, the distance to a closest cop is at least two). Similarly, an endpoint $v$ is bad if it is incident to a bad edge. An endpoint $v$ that is not bad is called threatening if it is incident to a threatening edge. Finally, $v$ is good if it is neither bad nor threatening.

We play the game against $\delta(G)-1$ cops. Since $G$ is connected, we may assume that all cops start at a single edge. Since the girth is at least 6 , the robber can start by going to an edge $u v$ that is at distance at least three from all the cops. No matter what they do in the next round, the distance between players is going to be at least two so both $u$ and $v$ are not going to be bad. We will show (by induction) that the robber has a strategy to guarantee that at every round of the game, when it is his turn to make a move, he is not caught and at least one endpoint is not bad.

Suppose that the robber occupying an edge $u v$ is not caught, at least one endpoint is not bad, and it is his turn to move. If both endpoints are good, the robber stands still. No matter what cops do in the next round, both endpoints might become threatening but are still not bad. Suppose then that $u$ is threatening or bad, and $v$ is threatening or good (in particular, there is no bad edge incident to $v$ ). At least one cop is within distance two from $u$ in $G-u v$ (the distance between an edge incident to $u$ and $u$ is 1 , etc.). Let us fix one such cop and call him dangerous. Since the girth is at least 6 , dangerous cop is at distance at least 3 from $v$ in $G-u v$. As a result, 


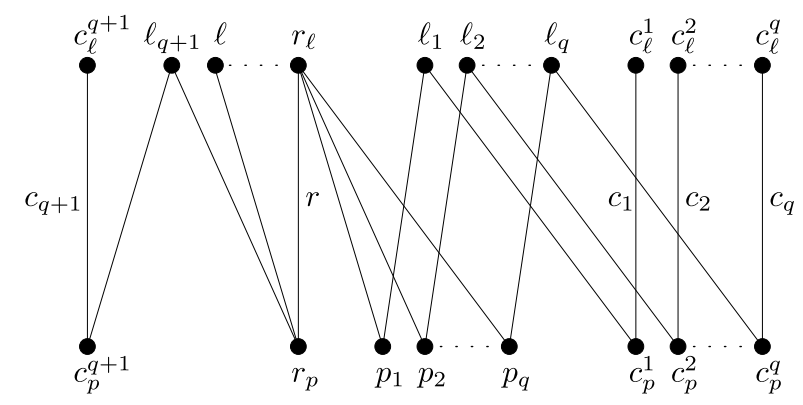

Figure 2: The 1st phase of the game played on $G(P)$.

dangerous cop does not make any edge incident to $v$ threatening. Moreover, every other cop can make at most one edge incident to $v$ threatening. Since there are $\delta(G)-2$ other cops (dangerous cop is disregarded) and the number of edges incident to $v$ is $\delta(G)-1$, there is at least one edge, say, $v w$ that is good. The robber moves from $u v$ to $v w$, and no matter what cops do in the next round $w$ is not going to be bad. The result holds.

Since $G(P)$ is $(q+1)$-regular, it follows from Lemma 4.1 that $\bar{c}(G(P)) \geq$ $q+1$, and so the family is $(1 / \sqrt{2})$-Meyniel extremal. On the other hand, from Theorem 2.1 we have that $\bar{c}(G(P)) \leq c(G(P))+1=q+2$, so only two values are possible. We eliminate one of them in the next theorem.

Theorem 4.2. Let $P:=P G(2, q)$ be the projective plane of order $q$. The incidence graph $G(P)$ of $P$ satisfies the following:

$$
\bar{c}(G(P))=q+1 .
$$

Proof. As we already mentioned, the lower bound of $q+1$ follows immediately from Lemma 4.1. It remains to show that $q+1$ cops can catch the robber. We will distinguish two phases of the game. In the first phase, we provide a strategy for cops so that one of them moves at distance one from the robber. (In fact, for that we could use the property that $c(G(P))=q+1$ and exploit an idea from the proof of Theorem 2.1 to couple the classic game with the game on edges. However, we provide an explicit strategy in order to avoid referencing to a result from [25] so that the argument presented here is self-contained.) During the second phase, the cop that is close to the robber keeps chasing him and the remaining cops make sure the bad guy is caught.

The cops start the game (and the first phase) by occupying $q+1$ independent edges $c_{i}=c_{p}^{i} c_{\ell}^{i}(i=1,2, \ldots, q+1)$-see Figure 2. Subscripts $p$ and 


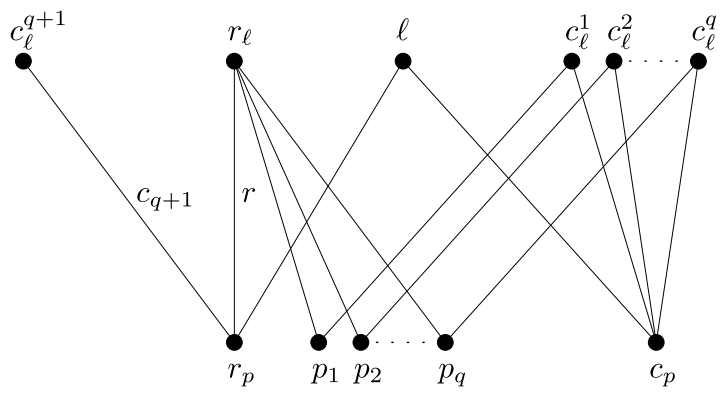

Figure 3: The 2nd phase of the game played on $G(P)$.

$\ell$ indicate that a vertex is a point and a line, respectively. We may assume that the robber goes to another independent edge $r=r_{p} r_{\ell}$; otherwise, he would be caught immediately. Let $p_{1}, p_{2}, \ldots, p_{q}$ be points on the line $r_{l}$ that are different than $r_{p}$ (recall that each line consists of $q+1$ points). For each $i=1,2, \ldots, q$, a pair of points $p_{i}, c_{p}^{i}$ belongs to a line $\ell_{i}$, and so $i$ th cop can be moved from $c_{p}^{i} c_{\ell}^{i}$ to $c_{p}^{i} \ell_{i}$. The remaining $(q+1)$ st cop has similar task. He is moved from $c_{p}^{q+1} c_{\ell}^{q+1}$ to $c_{p}^{q+1} \ell_{q+1}$, where $\ell_{q+1}$ is the line containing both $c_{p}^{q+1}$ and $r_{p}$. Regardless of what the robber does in the next round, $i$ th cop $(i=1,2, \ldots, q)$ moves to $p_{i} \ell_{i},(q+1)$ st cop moves to $r_{p} \ell_{q+1}$, and some cop occupies an edge incident to the one occupied by the robber-again, see Figure 2. This cop receives the task of chasing the robber till the end of the game. In order to keep the notation simple we may relabel cops so that $(q+1)$ st cop is chasing the robber.

Let $c_{p}$ be any point and $c_{\ell}^{1}, c_{\ell}^{2}, \ldots, c_{\ell}^{q}, \ell$ be $q+1$ lines this point belongs to. At the beginning of the second phase, the remaining $q$ cops move to the star centered at $c_{p}$; that is, $i$ th $\operatorname{cop}(i=1,2, \ldots, q)$ goes to $c_{p} c_{\ell}^{i}$-see Figure 3. (Recall that $G(P)$ is connected so it is possible.) Once they are at the desired positions, they wait till the robber and the chasing cop share a point; that is, say, the robber occupies an edge $r_{p} r_{\ell}$ and the chasing cop is at $r_{p} c_{\ell}^{q+1}$. A subtle but important observation is that we may assume that $r_{p}$ belongs to $\ell$. Indeed, if it were not the case, then $r_{p}$ would have to belong to a line $c_{\ell}^{i}$ for some $i$, as $r_{p}$ and $c_{p}$ share some line. But, since in the previous round the robber was at the edge $r_{p} c_{\ell}^{q+1}$ currently occupied by the chasing cop, the cop occupying $c_{p} c_{\ell}^{i}$ could have moved to $r_{p} c_{\ell}^{i}$ and the robber would have been already caught.

Assuming that $r_{p}$ belongs to $\ell$, we get that no $p_{i}(i=1,2, \ldots, q)$ belongs to $\ell$, since this would imply that there is a cycle $\left(p_{i}, \ell, r_{p}, r_{\ell}\right)$ of length 4 but the girth of $G(P)$ is 6 . Hence, for every $i=1,2, \ldots, q$ we have that 
$p_{i}$ belongs to $c_{\ell}^{j}$ for some $j=1,2, \ldots, q$, as $p_{i}$ and $c_{p}$ belong to some line. In the next round, the regular team of $q$ cops moves so that each point $p_{i}$ $(i=1,2, \ldots, q)$ is incident to a cop; the chasing cop keeps doing his job. No matter what the robber does in the next round, he will be trapped and the end of the game is near. The result holds.

\section{Random graphs}

Let us recall the classic model of random graphs that we study in this paper. The binomial random graph $\mathcal{G}(n, p)$ is defined as a random graph with vertex set $[n]=\{1,2, \ldots, n\}$ in which a pair of vertices appears as an edge with probability $p$, independently for each such pair. As typical in random graph theory, we shall consider only asymptotic properties of $\mathcal{G}(n, p)$ as $n \rightarrow \infty$, where $p=p(n)$ may and usually does depend on $n$. We say that an event in a probability space holds asymptotically almost surely (a.a.s.) if its probability tends to one as $n$ goes to infinity. (For more on random graphs, see, for example, [6, 18].)

Let us first briefly describe some known results on the classic cop number of $\mathcal{G}(n, p)$. (One can find much more on the related topics: for random $d$ regular graphs see [26], for random geometric graphs see [2, 5], and for the robber performing a random walk see [19, 20].) Bonato, Wang, and the third author investigated such games in $\mathcal{G}(n, p)$ random graphs, and their generalizations used to model complex networks with a power-law degree distribution (see [11]). From their results it follows that if $2 \log n / \sqrt{n} \leq p<$ $1-\varepsilon$ for some $\varepsilon>0$, then a.a.s.

$$
c(\mathcal{G}(n, p))=\Theta(\log n / p) .
$$

So Meyniel's conjecture holds a.a.s. for such $p$. Moreover, if $p=n^{-o(1)}$ and $p<1-\varepsilon$ for some $\varepsilon>0$, then a.a.s.

$$
c(\mathcal{G}(n, p))=(1+o(1)) \log _{1 /(1-p)} n .
$$

A simple argument using dominating sets shows that Meyniel's conjecture also holds a.a.s. if $p$ tends to one as $n$ goes to infinity (see [25] for this and stronger results). Somewhat surprisingly, it was shown by Łuczak and the third author [22] that for sparse random graphs the cop number has more complicated behaviour. It follows that a.a.s. $\log _{n} c\left(\mathcal{G}\left(n, n^{x-1}\right)\right)$ is asymptotically equal to the function $f(x)$ shown in Figure 4. Moreover, Bollobás, Kun, and Leader [7] showed that if $d=p(n-1)>(2+\varepsilon) \log n$ for some $\varepsilon>0$, then a.a.s. $c\left(\mathcal{G}\left(n, n^{x-1}\right)\right)=O(\sqrt{n} \log n)$. 


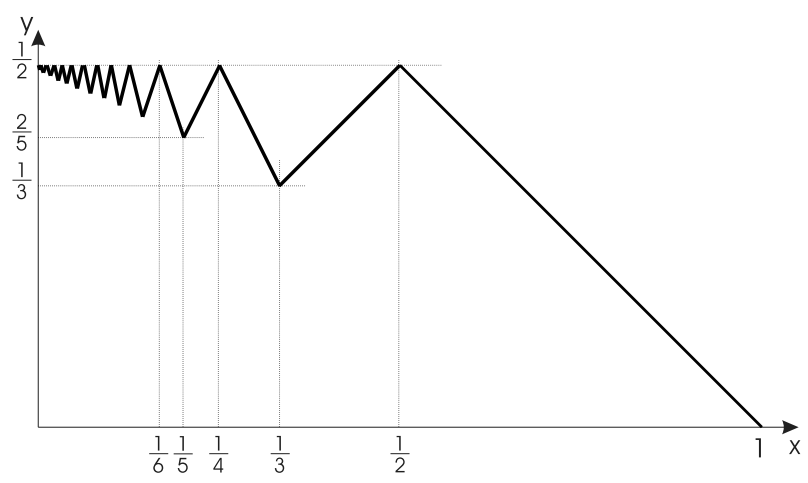

Figure 4: The "zigzag" function $f$.

From the above results, we know that Meyniel's conjecture holds a.a.s. for random graphs except perhaps when $n p=n^{1 /(2 k)+o(1)}$ for some $k \in \mathbb{N}$, or $n p=n^{o(1)}$. In [27], Wormald and the third author of this paper showed that the conjecture holds a.a.s. in $\mathcal{G}(n, p)$ provided that $n p>(1 / 2+\varepsilon) \log n$ for some $\varepsilon>0$. Note that Meyniel's conjecture is restricted to connected graphs, but $G \in \mathcal{G}(n, p)$ is a.a.s. disconnected when $n p \leq(1-\varepsilon) \log n$. Thus, the following equivalent statement of Meyniel's conjecture holds a.a.s. for $G \in \mathcal{G}(n, p)$ for all $p$ : if $G$ is connected then $c(G)=O(\sqrt{n})$.

In this paper, we investigate the edge cop number $\bar{c}(G)$. Since there is only a multiplicative constant factor difference between the two cop numbers (by Theorem 2.1), all known results for random graphs also hold for the edge cop number (up to a constant). In particular, Meyniel's conjecture holds a.a.s. for the edge cop number for $G \in \mathcal{G}(n, p)$ for all $p$. On the other hand, the asymptotic value of the classic cop number is known only for dense random graphs; that is, when $p=n^{-o(1)}$. We will show that in this case, the edge cop number is essentially two times smaller than its classic counterpart $($ see $(1))$.

Theorem 5.1. Let $p=p(n)$ be such that $p=n^{-o(1)}$ and $p<1-\varepsilon$ for some $\varepsilon>0$. Then, a.a.s.

$$
\bar{c}(\mathcal{G}(n, p))=\left(\frac{1}{2}+o(1)\right) \log _{1 /(1-p)} n
$$

Before we move to the proof of this theorem, let us mention an asymptotic behaviour of the logarithm used in the formula for the edge cop number, 
and state one general observation that will be useful in the proof. If $p$ is a constant smaller than 1 ,

$$
\log _{1 /(1-p)} n=\Theta(\log n)
$$

However, if $p=o(1)$ we have that

$$
\log _{1 /(1-p)} n=\frac{\log n}{-\log (1-p)}=(1+o(1)) \frac{\log n}{p}
$$

which tends to infinity faster than natural logarithm.

A set of vertices $S$ is a dominating set in $G$ if each vertex not in $S$ is joined to some vertex of $S$. The domination number of $G$, written $\gamma(G)$, is the minimum cardinality of a dominating set in $G$. A straightforward observation is that $c(G) \leq \gamma(G)$ (place a cop on each vertex of dominating set with minimum cardinality and catch the bad guy in the next round). Our goal here is to discover a similar bound for the edge cop number.

An edge cover of a set $S$ is a set of edges $C$ in a graph induced by $S$ such that each vertex in $S$ is incident with at least one edge in $C$. A minimum edge covering of $S$ is an edge covering of smallest possible size. The edge covering number of a set $S$ inducing a graph with no isolated vertex, written $\rho(S)$, is the size of a minimum edge covering of $S$. Finally, for a graph $G$ with no isolated vertex, let

$$
\xi(G)=\min \{\rho(S): S \in \mathcal{S}\}
$$

where $\mathcal{S}$ is a family of dominating sets satisfying the following property: for every set $S \in \mathcal{S}$ we have that a graph induced by $S$ has no isolated vertex. (The reason $G$ is assumed to have no isolated vertex is to make sure $\xi(G)$ is well defined.)

Now, we are ready to state a lemma that will be very useful in investigating dense random graphs. We present it as an independent result since it might be also applied to other families of graphs.

Lemma 5.2. Let $G=(V, E)$ be any connected graph. Then

$$
\bar{c}(G) \leq \xi(G)+1
$$

Note that the result is sharp, since for any $n \geq 4$ we have $\bar{c}\left(K_{n}\right)=2$ and $\xi\left(K_{n}\right)=1$.

Proof of Lemma 5.2. Let $S \in \mathcal{S}$ be a dominating set that yields $\xi(G)$ with a minimum edge cover $C$ of cardinality $c=\xi(G)$. The regular team of $c$ cops start at the edge cover $C$; one extra cop starts anywhere in the graph. 
The robber goes to an edge $e \in E$. Since every vertex of $S$ is covered, the robber is immediately caught if $e$ has at least one endpoint in $S$. Hence, we may assume that both endpoints of $e$ (say, $u$ and $v$ ) are not in $S$. Since $u$ is dominated by $S$ (recall that $S$ is a dominating set), there exists $u^{\prime} \in S$ such that $u u^{\prime} \in E$. Since $S$ induces no isolated vertex and $C$ covers all vertices of $S$, there is an edge $e^{\prime}$ in $C$ incident to $u^{\prime}$. The cop occupying $e^{\prime}$ moves to $u u^{\prime}$ and this cop will have a special task of chasing the robber till the end of the game. (If there are more cops incident to $u^{\prime}$, the decision of which cop should be chasing the robber can be made arbitrarily.) Now, the extra cop replaces the chasing cop, joins the regular team of $c$ cops, and moves to the edge $e^{\prime}$ of the edge cover $C$ that was previously occupied by the chasing cop. Since $G$ is connected, this task takes at most $|V(G)|-1$ steps. Once he is at the desired position, all cops look around and see the robber moving to an edge $e^{\prime \prime}$ with endpoints $x$ and $y$. As before, we may assume that neither endpoint is in $S$. The chasing cop moves as usual to secure one endpoint, say $x$. For the other endpoint, $y$, we take $y^{\prime} \in S$ such that $y y^{\prime} \in E$ and move a cop occupying an edge incident to $y y^{\prime}$ towards the robber. The robber is caught in the next round and the result holds.

Now we come back to the proof of the main result of this section.

Proof of Theorem 5.1. It follows from (1) that a.a.s. $c(\mathcal{G}(n, p))=(1+o(1))$. $\log _{1 /(1-p)} n$. By Theorem 2.1 we have $\bar{c}(G) \geq c(G) / 2$, and the lower bound follows immediately.

Let

$$
k=\log _{1 /(1-p)} n+2 \log _{1 /(1-p)} \omega,
$$

where $\omega=\omega(n)=p^{-1} \log n$. Clearly, $\omega p=\log n \rightarrow \infty$ as $n \rightarrow \infty$, but note also that $\log _{1 /(1-p)} \omega=o(k)$ so, in fact, $k=(1+o(1)) \log _{1 /(1-p)} n$. Indeed, if $p=\Omega(1)$, then $\log _{1 /(1-p)} \omega=\Theta(\log \log n)=o(\log n)=o(k)$. On the other hand, for $p=o(1)$ we have $\omega=n^{o(1)}$ and

$$
\log _{1 /(1-p)} \omega=(1+o(1)) \frac{\log \omega}{p}=o\left(\frac{\log n}{p}\right)=o(k) .
$$

For the upper bound we are going to use Lemma 5.2. Our goal is to find a set that is a.a.s. dominating and has small edge cover. Fix any set $X$ of cardinality $k$. First of all, we will show that a.a.s. there exists a set $Y \subseteq X$ of cardinality $\log _{1 /(1-p)} n+\log _{1 /(1-p)} \omega=k-\log _{1 /(1-p)} \omega$ which has a perfect matching. In order to do it, we consider the following process. Select any vertex $x_{1}$ and expose all edges from $x_{1}$ to other vertices of $X$. If at least one edge is found, select any neighbour $x_{2}$ of $x_{1}$ and pick another vertex $x_{3} \in X \backslash\left\{x_{1}, x_{2}\right\}$. This time we expose all edges from $x_{3}$ to $X \backslash\left\{x_{1}, x_{2}, x_{3}\right\}$ 
with a hope that at least one edge is discovered and the process can be continued. The only reason for the process to terminate at a given round is when no edge is found. We will show that a.a.s. the process does not stop before all but $r:=\log _{1 /(1-p)} \omega$ vertices are matched. Indeed, the probability the process does not stop earlier is equal to

$$
\begin{aligned}
\left(1-(1-p)^{k-1}\right) & \left(1-(1-p)^{k-3}\right) \cdot \ldots \cdot\left(1-(1-p)^{r+1}\right) \\
& =\prod_{i=r / 2}^{k / 2-1}\left(1-(1-p)^{2 i+1}\right) \\
& =\exp \left(-(1+o(1))(1-p) \sum_{i=r / 2}^{k / 2-1}(1-p)^{2 i}\right) \\
& =\exp \left(-(1+o(1))(1-p) \frac{\left((1-p)^{2}\right)^{r / 2}-\left((1-p)^{2}\right)^{k / 2}}{1-(1-p)^{2}}\right) \\
& =\exp \left(-(1+o(1)) \frac{1-p}{1-(1-p)^{2}} \omega^{-1}\right) \\
& =\exp \left(-O\left((p \omega)^{-1}\right)\right),
\end{aligned}
$$

which tends to one, since $p \omega$ tends to infinity as $n \rightarrow \infty$.

Second of all, we will show that a.a.s. $Y \subseteq X$ dominates all vertices of $V \backslash X$. (Note that edges between $Y$ and $V \backslash X$ are not exposed yet. However, edges between $Y$ and $X \backslash Y$ are already exposed and so we cannot investigate if $X \backslash Y$ is dominated by $Y$ or not.) Since $|Y|=\log _{1 /(1-p)} n+\log _{1 /(1-p)} \omega$ and $|X|=\log _{1 /(1-p)} n+2 \log _{1 /(1-p)} \omega$, this probability is equal to

$$
\begin{aligned}
\left(1-(1-p)^{|Y|}\right)^{n-|X|} & \geq 1-(n-|X|)(1-p)^{|Y|} \\
& \geq 1-n(1-p)^{\log _{1 /(1-p)} n+\log _{1 /(1-p)} \omega} \\
& =1-1 / \omega
\end{aligned}
$$

which tends to one as $n \rightarrow \infty$.

Third of all, we will show that a.a.s. the set $X \backslash Y$ induces a graph with no isolated vertex. (Note that edges within $X \backslash Y$ are not exposed yet.) The probability that a given vertex in $X \backslash Y$ is isolated is $(1-p)^{|X \backslash Y|-1}=O\left(\omega^{-1}\right)$. Hence, the expected number of isolated vertices is

$$
O\left(\omega^{-1} \log _{1 /(1-p)} \omega\right)=O\left(\frac{\log \omega}{\log n}\right)=o(1),
$$

and the result holds by Markov's inequality. 
Putting the three claims together we will get the upper bound. It follows that a.a.s. $X$ is a dominating set (since $Y \subseteq X$ dominates $V \backslash X$ a.a.s.). Moreover, a.a.s.

$$
\rho(X) \leq \rho(Y)+\rho(X \backslash Y) \leq \frac{|Y|}{2}+|X \backslash Y|=\frac{1}{2} \log _{1 /(1-p)} n+\frac{3}{2} \log _{1 /(1-p)} \omega,
$$

since a.a.s. $Y$ has a perfect matching, and $X \backslash Y$ induces no isolated vertex so one can simply take any incident edge for each vertex of $X \backslash Y$. Hence, a.a.s.

$$
\xi(G) \leq \rho(X) \leq(1 / 2+o(1)) \log _{1 /(1-p)} n .
$$

The proof of the upper bound follows, since $\bar{c}(G) \leq \xi(G)+1$ by Lemma 5.2.

The upper bound presented in the proof of the previous theorem can be easily extended to sparser graphs. As we already mentioned, the reason we focus on $p=n^{-o(1)}$ is because for sparser graphs the bounds for the classic cop number are not tight and so there is no easy argument to get a matching lower bound.

Moreover, let us mention that it is known that a.a.s. $\mathcal{G}(n, p)$ has a perfect matching, provided that $p$ is above the threshold for disappearing isolated vertices (see, for example, $[6,18]$ ). This result could be used to show that the set $X$ in the proof of the previous theorem has a perfect matching a.a.s. (of course, some rescaling is need, since the number of vertices is not $n$, and so the argument is more subtle). We presented an independent simple proof to make the argument self-contained.

\section{Planar graphs}

As was already mentioned in the introduction, Aigner and Fromme [1] proved that any connected planar graph $G$ satisfies $c(G) \leq 3$. Here we prove an analogous result for the new variant of the game.

Theorem 6.1. Let $G$ be any connected planar graph. Then

$$
\bar{c}(G) \leq 3 .
$$

Before we move to the proof, we note that the bound is best possible, since one can easily verify that the edge cop number of the graph on Figure 5 is at least 3 (and consequently, exactly 3 ).

The proof is a direct adaptation of the approach taken by Aigner and Fromme [1]. We say that a cop $c$ controls a path $P$ if whenever the robber 


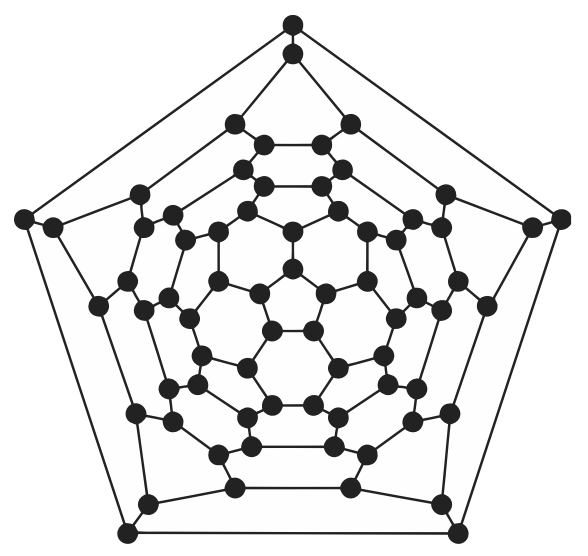

Figure 5: A plane representation of the soccer ball. (Each pentagon is surrounded by 5 hexagons.)

tries to cross $P$, then he is caught by $c$ on his responding moves. First we prove an auxiliary result showing that one cop suffices to control any shortest path in an arbitrary connected graph.

Lemma 6.2. Let $G$ be any graph, $u, v \in V(G), u \neq v$, and $P=(u=$ $v_{0}, v_{1}, \ldots, v_{s}=v$ ) be a shortest path between $u$ and $v$. A single cop can control $P$ after a finite number moves.

The idea of the proof of Theorem 6.1 is to divide the pursuit into stages. In stage $i$, we assign to the robber $r$ a certain subgraph $G_{i}$, the robber's territory, which contains all edges which $r$ may still safely enter, and we show that, after a finite number of cop-moves, $G_{i}$ is reduced to $G_{i+1} \subsetneq G_{i}$. Eventually, there is no safe edge left for the robber and the game ends. In each iteration, at most two shortest paths in $G_{i}$ must be controlled. Due to Lemma 6.2, this requires one cop per path, and the third cop moves to control another shortest path in $G_{i}$. See [1] for the details of the proof.

It remains to prove the auxiliary lemma.

Proof of Lemma 6.2. We will deduce Lemma 6.2 from a similar result from [1]. In the following definition we assume that the robber and cops play on vertices. We say that a set of cops guard (vertex-wise) a path $P$ from a set of robbers if whenever a robber steps onto $P$, he steps onto a cop or is caught by a cop on his responding move. It was shown in [1] that one cop suffices to guard any shortest path from one robber. In order to achieve it, a projection of the vertex $r$ occupied by the robber on the path 
$P$ was introduced. This projected vertex, $s$, was called the shadow of the robber. The cop strategy is simply to catch the shadow of the robber and keep following it. We will show that this is also the case for the new variant of the game.

We start the game played on edges with the cop going to any edge $c$ on the path $P=\left(v_{0}, v_{1}, \ldots, v_{s}\right)$ and $r$ goes anywhere. At any point of the game played on edges and for any player $p$ occupying an edge $e$ (regardless whether it is a cop or the robber), we couple $p$ with two vertex players $p(1)$ and $p(2)$ which are the endpoints of $e$. Every move of $p$ induces a move of both $p(1)$ and $p(2)$. In particular, every edge-move of $r$ induces a vertexmove of $r(1)$ and $r(2)$. Clearly, when the robber moves, the corresponding vertices $r(1)$ and $r(2)$ move to their neighbours and the distance between them is one. The key observation is that the corresponding shadows $s(1)$ and $s(2)$ move to neighbours or remain still, and the distance between $s(1)$ and $s(2)$ is always at most one. In order to show that a cop $c$ controls $P$, we prove that $c(1)$ and $c(2)$ can guard $P$ from $r(1)$ and $r(2)$ by catching their shadows $s(1)$ and $s(2)$, respectively. (If $s(1)=s(2)=s$, then $c(1)$ catches $s$ and $c(2)$ occupies any neighbour of $s$.)

During the first phase of the game, the cop plays so that $c(1)$ catches $s(1)$ as in the classic version of the game. If $s(2)=s(1)$ or $s(2)=c(2)$, then the path is already guarded. Hence, due to the symmetry, we may suppose that $c(1)=s(1)=v_{i}, s(2)=v_{i+1}$, and $c(2)=v_{i-1}$. If the robber stands still, we can easily catch his shadows: the cop moves to get $c(1)=v_{i+1}=s(2)$ and $c(2)=v_{i}=s(1)$, and the path is guarded, since we may always swap $c(1)$ and $c(2)$, if needed. If the robber moves so that $s(1)=v_{i-1}$ and $s(2)=v_{i}$, the cop does not move and the desired property holds. If the robber moves so that $s(1)=s(2)$, the cop can respond to finish the task as well. The only reasonable move for the robber is to move so that $s(1)=v_{i+1}$ and $s(2)=v_{i+2}$ (if such a move exists), but the cop can chase the robber and move towards $v_{s}$ with no problem. However, since the path is finite, the robber has to finally give up and at some point of the game both shadows are guarded.

It remains to show that the cop has a strategy to keep following the shadows. Due to the symmetry, we may assume that $s(1)$ is always as close to $v_{0}$ as $s(2)$. Suppose first that $s(1)=s(2)=c(1)=v_{i}$ and $c(2)=v_{i+1}$ (the case $c(2)=v_{i-1}$ is symmetric and so omitted). If the robber stands still or moves so that $s(1)=s(2)=v_{i+1}$ or $s(1)=v_{i}$ and $s(2)=v_{i+1}$, the cop does not move. If the robber moves so that $s(1)=s(2)=v_{i-1}$ or $s(1)=v_{i-1}$ and $s(2)=v_{i}$, then the cop moves towards $v_{0}$. Suppose then that $s(1)=c(1)=v_{i}$ and $s(2)=c(2)=v_{i+1}$. If the robber moves so that 
$s(1)=v_{i-1}$ and $s(2)=v_{i}$ (or $s(1)=v_{i+1}$ and $s(2)=v_{i+2}$ ), the cop moves towards $v_{0}$ (or $v_{s}$, respectively). If the robber moves so that $s(1)=s(2)$, the cop remains still. Hence, the cop has a strategy to guard the path $P$ and the proof is finished.

\section{Open problems}

We finish the paper with a few open problems and future directions.

- As we already mentioned, the upper bound for $\bar{c}(G)$ in Theorem 2.1 is sharp. Graphs with $c(G)=1$ are characterized (see [23]) and in this paper we characterize graphs with $\bar{c}(G)=1$. Hence, there is a characterization of graphs with $c(G)=1$ and $\bar{c}(G)=2$. Is there any graph with $\bar{c}(G)=c(G)+1>2$ ? If the answer is yes, it should be the case that these graphs have some structure that should be relatively easy to investigate. We leave it as an open problem to characterize graphs with this property.

- The lower bound for $\bar{c}(G)$ in Theorem 2.1 is equivalent to $c(G) \leq$ $2 \bar{c}(G)$. However, it follows from Corollary 3.5 that there is no graph $G$ with $\bar{c}(G)=1$ and $c(G)=2$. We provided an example with $c(G)=3$ and $\bar{c}(G)=2$ but no example is known for which $c(G)=2 \bar{c}(G)$. We feel that there is a small improvement possible and, in fact, $c(G) \leq$ $2 \bar{c}(G)-1$ for any connected graph. On the other hand, the result for random graphs implies that the multiplicative factor of 2 cannot be improved.

- Let

$$
A=\left\{\frac{\bar{c}(G)}{c(G)}: G \text { is a connected graph }\right\} .
$$

It follows from Theorem 2.1 that the closure of $A, \bar{A}$, satisfies the following

$$
\bar{A} \subseteq B:=\left[\frac{1}{2}, 1\right] \cup\left\{2, \frac{3}{2}, \frac{4}{3}, \ldots\right\},
$$

but all we showed is that $\{1 / 2,2 / 3,1,2\} \subseteq \bar{A}$. Is it true that $A=B$ ?

- We characterized graphs with the edge cop number one. Is there a characterization of graphs with $\bar{c}(G)=k$ for some $k \in \mathbb{N}$ ?

- We concentrated on dense random graphs; that is, when $p=n^{-o(1)}$. As we mentioned earlier, the asymptotic value of the classic cop number is not known for sparse random graphs. However, it still might be possible that the edge cop number is essentially two times smaller 
than its classic counterpart; that is, it might be possible to show that a.a.s. the ratio $\bar{c}(\mathcal{G}(n, p)) / c(\mathcal{G}(n, p))$ is equal to $1 / 2+o(1)$ without investigating the behaviour of $c(\mathcal{G}(n, p))$.

\section{References}

[1] M. Aigner and M. Fromme, A game of cops and robbers. Discrete Applied Mathematics 8 (1984), 1-12. MR0739593

[2] N. Alon and P. Prałat, Chasing robbers on random geometric graphsan alternative approach, preprint.

[3] B. Alspach, Sweeping and searching in graphs: A brief survey. Matematiche 59 (2006), 5-37. MR2243023

[4] W. Baird and A. Bonato, Meyniel's conjecture on the cop number: A survey. Journal of Combinatorics 3 (2012), 225-238. MR2980752

[5] A. Beveridge, A. Dudek, A. Frieze, and T. Müller, Cops and robbers on geometric graphs. Combinatorics, Probability and Computing 21(6) (2012), 816-834. MR2981156

[6] B. Bollobás, Random Graphs. Second Edition. Cambridge University Press, Cambridge, 2001. MR1864966

[7] B. Bollobás, G. Kun, and I. Leader, Cops and robbers in a random graph. Journal of Combinatorial Theory Series B 103 (2013), 226-236. MR3018067

[8] A. Bonato and A. Burgess, Cops and robbers on graphs based on designs. Journal of Combinatorial Designs 21 (2013), 359-418. MR3085764

[9] A. Bonato, G. Kemkes, and P. Prałat, Almost all cop-win graphs contain a universal vertex. Discrete Mathematics 312 (2012), 1652-1657. MR2901161

[10] A. Bonato and R. Nowakowski, The Game of Cops and Robbers on Graphs. American Mathematical Society, 2011. MR2830217

[11] A. Bonato, P. Prałat, and C. Wang, Network security in models of complex networks. Internet Mathematics 4 (2009), 419-436.

[12] E. Chiniforooshan, A better bound for the cop number of general graphs. Journal of Graph Theory 58 (2008), 45-48. MR2404040 
[13] P. Frankl, Cops and robbers in graphs with large girth and Cayley graphs. Discrete Applied Mathematics 17 (1987), 301-305. MR0890640

[14] F. V. Fomin and D. Thilikos, An annotated bibliography on guaranteed graph searching. Theoretical Computer Science 399 (2008), 236-245. MR2419780

[15] A. Frieze, M. Krivelevich, and P. Loh, Variations on cops and robbers. Journal of Graph Theory 69 (2012), 383-402. MR2979296

[16] C. Godsil and G. Royle, Algebraic Graph Theory. Springer, New York, 2001. MR1829620

[17] G. Hahn, Cops, robbers and graphs, Tatra Mountain Mathematical Publications 36 (2007), 163-176. MR2378748

[18] S. Janson, T. Łuczak, and A. Ruciński, Random Graphs. Wiley, New York, 2000. MR1782847

[19] A. Kehagias and P. Prałat, Some remarks on cops and drunk robbers. Theoretical Computer Science 463 (2012), 133-147. MR2992504

[20] A. Kehagias, D. Mitsche, and P. Prałat, Cops and invisible robbers: The cost of drunkenness. Theoretical Computer Science 481 (2013), 100-120. MR3037800

[21] L. Lu and X. Peng, On Meyniel's conjecture of the cop number. Journal of Graph Theory $\mathbf{7 1}$ (2012), 192-205. MR2965383

[22] T. Euczak and P. Prałat, Chasing robbers on random graphs: Zigzag theorem. Random Structures and Algorithms 37 (2010), 516-524. MR2760362

[23] R. Nowakowski and P. Winkler, Vertex to vertex pursuit in a graph. Discrete Mathematics 43 (1983), 230-239. MR0685631

[24] P. Prałat, Almost all $k$-cop-win graphs contain a dominating set of cardinality $k$, preprint.

[25] P. Prałat, When does a random graph have constant cop number? Australasian Journal of Combinatorics 46 (2010), 285-296. MR2598712

[26] P. Prałat and N. C. Wormald, Meyniel's conjecture holds for random $d$-regular graphs, preprint.

[27] P. Prałat and N. C. Wormald, Meyniel's conjecture holds for random graphs, preprint. 
[28] A. Quilliot, Jeux et pointes fixes sur les graphes. Thèse de 3ème Cycle. Université de Paris VI, 1978, 131-145.

[29] A. Quilliot, Problèmes de jeux, de point Fixe, de connectivité et de represésentation sur des graphes, des ensembles ordonnés et des hypergraphes. Thèse d'Etat. Université de Paris VI, 1983, 131-145.

[30] A. Scott and B. Sudakov, A bound for the cops and robbers problem. SIAM J. of Discrete Math 25 (2011), 1438-1442. MR2837608

ANDRZEJ DUDEK

Department of Mathematics

WESTERn Michigan University

KaLAmazoO, MI

USA

E-mail address: andrzej.dudek@wmich.edu

PrZemystaW Gordinowicz

Institute OF Mathematics

TEChNiCAL UnIVERSITY OF LODZ

ŁóDŹ

Poland

E-mail address: pgordin@p.lodz.pl

Pawee Pratat

Department of Mathematics

RYERSON UNIVERSITY

TORONTO, ON, M5B 2K3

CANADA

E-mail address: pralat@ryerson.ca

Received January 5, 2013 